International Journal of Applied Linguistics \& English Literature

ISSN 2200-3592 (Print), ISSN 2200-3452 (Online)

Vol. 1 No. 5; September 2012 [Special Issue on General Linguistics]

\title{
The New Development of the Study of Discourse Anaphora -----Review of Discourse Anaphora: A Cognitive-Functional Approach
}

\author{
Meixia Li (Corresponding author) \\ School of English Language, Literature and Culture, Beijing International Studies University \\ No. 1 Nanli, Dingfuzhuang, Chaoyang District, Beijing, 100024, China \\ Tel: 86-10-6574-2856 E-mail: lmx595@yahoo.com.cn
}

Received: 05-05- 2012

doi:10.7575/ijalel.v.1n.5p.14
Accepted: 07-07- 2012

Published: 03-09- 2012

The research is financed by Funding Project for Academic Human Resources Development in Institutions of Higher Learning Under the Jurisdiction of Beijing Municipality No. PHR201006131 \& Research Planning Fund Project of China Ministry of Education, the Humanities and the Social Sciences No. 12 YJA740039

\section{Introduction}

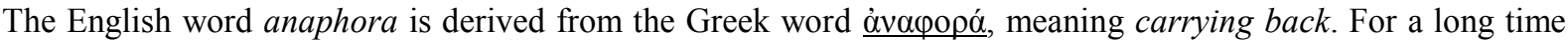
anaphora has been the object of research in a wide range of disciplines, such as rhetoric, philosophy, theoretical linguistics and so on. A great number of remarkable achievements have been made in these fields. In the 1970's there was a "discourse turn" in the domain of the humanities and the social sciences, which marked the birth and flourishing of such cross-disciplines as psycholinguistics, computational linguistics, cognitive linguistics, corpus linguistics, discourse studies and so on, and which also paved the way for the turn of the study of anaphora from focusing on intrasentential anaphora to intersentential anaphora. Intrasentential anaphora refers to the relationship between a pronoun and its antecedent being contained within one sentence, while intersentential anaphora can also be called discourse anaphora, which refers to "the relationship between a pronoun and its antecedent earlier in the discourse" (Clark \& Parikh, 2006, p. 1). From the late $20^{\text {th }}$ century on, discourse anaphora has become one of the hot topics in several fields such as psychology, cognitive science, artificial intelligence, etc. Many fruitful research results (i.e. Huang, 2002; Clark \& Parikh, 2006, etc.) have been obtained. Ming-Ming Pu's monograph Discourse Anaphora: A Cognitive-Functional Approach, published by LINCOM $\mathrm{GmbH}$ in Muenchen, Germany in 2011 is another important work of the study of discourse anaphora. In this book, the author first proposes a cognitive-functional model to account for how the construction of mental structures determines the use and resolution of discourse anaphora. Afterwards he does a comparative quantitative study of both English and Chinese empirical and text data, which demonstrates that on the one hand the occurrence and distribution of discourse anaphora is more universal in nature than language-specific, and on the other hand that the proposed model is adequate, feasible and workable. This book suits such readers as university teachers, graduate students and researchers who are interested in the study of anaphora, cross-linguistic studies, discourse analysis, and language teaching and learning. In the following I shall review each chapter and then offer my evaluation.

\section{Chapter overview}

This monograph contains 7 chapters. In the introductory chapter, the author first situates discourse anaphora in a new perspective. Discourse anaphora is not held as a static product or entity linked to its linguistic antecedent in a text but as a manifestation of cognitive processes of memory and attention, and of building discourse coherence and maintaining local and global topics, along with the tacit cooperation between speakers and hearers. Against this background this chapter aims to construct a cognitive-functional model to account for the use and resolution of discourse anaphora. Then, the scope of the book, the categorization of anaphora and the overview of the book are given sequentially. This introduction sets the anchoring point, establishing the structure of the book and providing readers with contextual information so that they have the necessary knowledge about the present research topic. 
International Journal of Applied Linguistics \& English Literature

ISSN 2200-3592 (Print), ISSN 2200-3452 (Online)

Vol. 1 No. 5; September 2012 [Special Issue on General Linguistics]

Drawing on prior research in diverse yet related fields such as psychology, neuroscience and linguistics, the second chapter discusses the role that memory and attention mechanisms play in information processing, followed by exploration of how memory and attention mechanisms constrain language production and comprehension in general, and reference tracking in discourse in particular. After the theoretical discussion, the author states that discourse processing is a collaborative process, in which the speaker makes an effort to facilitate the hearer's access to the referent with ease so that the hearer can build his /her mental representation of discourse congruent with his/her own by the usage of anaphora. This chapter serves as the theoretical foundation for the following study.

The third chapter presents a cognitive-functional model of discourse anaphora. It first argues that discourse anaphora is a hearer-oriented process, during which the speaker constantly assesses the activation status of referents in the hearer's mind and chooses specific anaphora to code the referents accordingly. Then, the author discusses how the two important factors -- topicality and thematic coherence -- modulate the activation level and attention activity of the referent during discourse processing, making the referent more or less accessible at the moment of utterance. Next, the author proposes a cognitive-functional model which explicates the relationship among cognitive activities, mental structure building, and discourse anaphora. Finally, the author holds a discussion of some of the most important functional and cognitive theories on anaphora, such as the Activation Model, the Referential Distance Model, Centering Theory, Accessibility Theory, and the Attention Model, critically pointing out the strengths and weaknesses of each, and stating that his proposed model differs significantly from others.

By presenting two experimental studies, Chapter Four is devoted to the validity of the proposed cognitive-functional model of discourse anaphora. Specifically speaking, the experiments are intended to realize three goals. The first goal is to examine whether the speaker's anaphoric choice in discourse production would be directly influenced by a disruption in cognitive activities. The second goal is to investigate whether the speaker's anaphoric choice would be affected by units of memory representations corresponding to text structures of episodes or paragraphs in oral and written discourse. The third goal is to find out what interrupts thematic coherence in the flow of information and whether different choices of anaphors are the result of major and minor thematic discontinuities in discourse. The two experiments are employed to fulfill the three goals. The first experiment examines the connection between cognitive mechanisms and discourse anaphora, specifically, the effect of major disruptions in the flow of information on the use of anaphora. The second experiment focuses on the effect of minor thematic discontinuities in the cognitive accessibility of referents and hence the use of anaphora. In the two experiments, the stimulus material is from a children's picture storybook entitled "Here Comes Alex Pumpernickel", which contains altogether eight episodes, three of which were adapted for the experiment. The participants are native speakers of both English and Chinese. They all completed the experiment in their own native language. In the first experiment there are forty participants, twenty native speakers of Canadian English and twenty native speakers of Mandarin Chinese. Ten of the Chinese speakers and thirteen of the English speakers are female. They are all graduate students at the university of Alberta, Canada. In this experiment the twenty-four pictures chosen from the three episodes of the above-mentioned storybook were made into a black and white video program, which could be viewed as a cartoon picture sequence from a computer screen. There are four experimental conditions: Chinese Even, Chinese Odd, English Even, and English Odd. The participants were randomly assigned to each of the conditions in equal numbers, namely ten participants for each condition. The tasks for each participant include both on-line and recall; and participants were asked to produce oral and written narratives, which were tape-recorded and later on transcribed by the experimenter. Then a detailed analysis of the data is carried out and the results confirm the Working Hypothesis 1 the author proposed at the beginning and prove the feasibility of the author's CF (cognitive-functional) model. In the second experiment there are also forty participants, twenty of whom are native speakers of (Mandarin) Chinese from Huazhong University of Finance and Economics, China, and another twenty of whom are native speakers of (American) English from Northern State University, USA. All of the participants are undergraduates and half of them are female. They are required to do two tasks: spoken and written, each of which is done individually. In the spoken task participants were asked to thumb through the picture book and simultaneously produce a spoken narrative, which were tape-recorded and later on transcribed. After the spoken description, participants were asked to write down the entire story they had just narrated, and the written data was collected immediately. Afterwards the author analyzed the data in terms of the purpose of the experiment, giving strong support to her proposal and her CF model. All in all, 
International Journal of Applied Linguistics \& English Literature

ISSN 2200-3592 (Print), ISSN 2200-3452 (Online)

Vol. 1 No. 5; September 2012 [Special Issue on General Linguistics]

the two experiments further indicate that discourse anaphora is governed by cognitive mechanisms of memory and attention, with the modulation of thematic coherence and topicality.

Chapter Five investigates whether the proposed model can account for discourse anaphora in naturally occurring, character-rich and plot-complex written narrative texts, and whether the major anaphoric patterns obtained from the two experiments can be found in complex written narrative texts. The study lends further support to the statement made in Chapter Three, that is, the cognitive constraints of memory and attention underlying reference tracking can also apply to naturally occurring and structurally complex written narratives, and the analyses of both contemporary English and Chinese short stories demonstrate that similar distributional patterns of anaphora are used between literary writers of the short stories and student writers in the experimental studies.

Based on the evidence from the experimental studies and text analysis, Chapter Six argues that the use of zero anaphora, rather than being constrained by language-specific characteristics, is also governed by the same cognitive-functional principle underlying anaphora tracking. With respect to the fact that zero anaphora occurs about twice as frequently in Chinese as it does in English discourse, the author states that the difference is mainly due to the definition and classification of "clause", and the determining factor that influences the use of zero anaphora in Chinese and English discourse is the sustained attentional effort on a referent that is maintained in a micro-unit of maximum thematic coherence. This chapter also deals with three types of maximum thematic continuity: topic continuity, action/event continuity, and condensed continuity, which, the author argues, are responsible not only for the occurrence and distribution of zero anaphora but also for the asymmetry between zero subject and zero object in both languages. In this chapter, Topic Chain, "in which the topic is almost invariably coded by zero subject, although zero objects occur occasionally" (Ming-Ming Pu, 2011, p. 161), and which is the characteristic feature of Chinese, is also taken up and a quite different conclusion is drawn. The author finds that topic chain can also be used in English discourse. The chapter further explores thematic discontinuity that inhibits the use of zero anaphora. In the end, a fill-in-the-blanks study was used "to test the psychological reality of thematic continuity and discontinuity in discourse processing, and to determine whether such continuity and discontinuity govern the use of covert versus overt referential forms" (Ming-Ming Pu, 2011, p. 207). The study reveals that native speakers of both English and Chinese follow the same general rules of anaphor use put forward by the author's CF model, and their choice of anaphora is in most cases consistent with what the authors of narratives do.

The last chapter sums up the major findings of the study in detail, and presents the necessity for further studies.

\section{Evaluation}

The study of anaphora can be classified into two sorts: one is the study of intrasentential anaphora (specifically, binding relations); the other is the study of intersentential anaphora (or discourse anaphora). "The former attracted attention in the 1960s and is one of the central topics in generative syntax and semantics, but also in current typological studies. The latter has been studied extensively since the early 1990s within computational linguistics, discourse representation theory, and functional approaches such as centering theory"(Reuland et al., 2011).

It is obvious that Ming-Ming Pu's research belongs to the latter. The book written by Ming-Ming Pu makes an important and innovative contribution to the study of anaphora, specifically discourse anaphora. Traditionally anaphora is defined as involving a "linguistic element which refers back to another linguistic element $\Leftrightarrow$ antecedent) in the coreferential relationship, i.e. the reference of an anaphora can only be ascertained by interpreting its antecedent" (Bussmann, 2000, p. 23), yet in this book, anaphora is regarded as "a process where references are managed in a developing discourse to maintain discourse topics and achieve coherence" (Ming-Ming Pu, 2011, p. 4). This definition has three merits. First, by using such expressions as "a process" and "a developing discourse", this definition emphasizes the dynamics of references; second, by the usage of the word "manage", this definition stresses the role of man's cognition or cognitive mechanism in the management of reference; third, "to maintain discourse topics and achieve coherence" clearly demonstrates the function of anaphora in a developing discourse. Thus it can be stated that anaphora is inextricably intertwined with cognition, function and dynamics, which not only enlarges the scope of anaphora, but also presents us a complete fresh perspective towards anaphora.

Then, rather than assuming that the processing of discourse anaphora is a static, textual, individual, linear, and isolated process, this study holds that it is a cognitive, interactional, hierarchical and contextual process. The proposal of this idea is an integration of the previous approaches and the author's own reconstruction. Actually

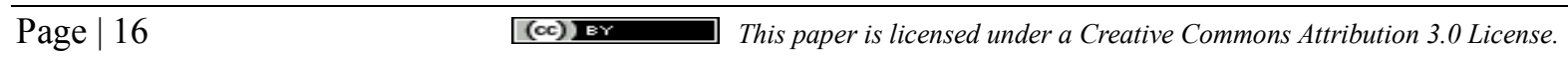


International Journal of Applied Linguistics \& English Literature

ISSN 2200-3592 (Print), ISSN 2200-3452 (Online)

Vol. 1 No. 5; September 2012 [Special Issue on General Linguistics]

in the domain of discourse anaphora four main theoretical models have already existed. They are: (1) the topic continuity model, (2) the hierarchical model, (3) the cognitive model, (4) a neo-Gricean pragmatic analysis of discourse anaphora (Huang, 2000). It can be easily seen that in addition to drawing on her predecessors, Ming-Ming Pu also contributed to the existing body of knowledge of discourse anaphora. Her effort is to delineate the universality of the principles governing the use and interpretation of anaphora.

Apart from that, rather than fixing her attention on anaphora and text information, the author establishes the relationship among the cognitive mechanism of memory and attention, and discourse coherence and anaphora, which unveils the true reason that leads to the use and resolution of discourse anaphora, that is, the use and resolution of anaphora is not a linguistic matter, rather it is a manifestation of the interaction between cognition and function. It can be further inferred that cognitive mechanism of memory and attention is primary and the linguistic element of anaphora is secondary.

Additionally, rather than simply generalizing the research on the use of anaphora, this study makes a deep investigation into what exactly contributes to the easy accessibility of referents. According to the experiment, this study finds that the two factors ------ topicality and thematic coherence in discourse processing determine the degree of accessibility of referents.

And, rather than merely relying upon qualitative English data, the author adopts both English and Chinese empirical and text data and analyzes them quantitatively and qualitatively. Here, what deserves attention is that the author does not just focus on the linguistic analysis of both English and Chinese narratives; on the contrary, by analyzing the spoken and written data obtained from two experiments and the major patterns of anaphora and their significance, the author intends to test her CF model and the particular hypotheses derived from it. The design of the experiments is rational. The selection of the material, the method and the procedure fit in with the purpose of the study. So the results of the study are credible.

Finally this study sheds new light on the issue of topic chain, with which zero anaphora is closely connected. It has been traditionally assumed that topic chain is a device unique to Chinese, because it is universally held that Chinese is a topic-oriented language. By contrast, the present study argues that topic chain can often be employed in English discourse when topicality and coherence are guaranteed, and so the phenomenon of topic chain is more language-general. This argument may subvert our long-held, deep-rooted thinking that topic chain is only found in Chinese.

All in all, this study offers us an extensive overview of the previous theories of the study of anaphora, as well as a well-established theoretical framework for the interpretation of discourse anaphora. It also presents some new thought-provoking ideas concerned with the processing of discourse anaphora and opens up more possibilities for further research.

In spite of the above-mentioned strengths, in this monograph, there are a few points which need reconsidering. It is usually the case that in a research a literature review should appear early, because "A literature review is a body of text that aims to review the critical points of current knowledge including substantive findings as well as theoretical and methodological contributions to a particular topic"(Wikipedia). Nevertheless, in this book, the author does not present a literature review first; instead, in Chapter Three, she first puts forward her model and then gives a critical assessment of the previous theories of the study of anaphora, which serves as a literature review. But in the reviewer's opinion, this critical literature review should be placed in an independent chapter and be presented earlier. A literature review can facilitate readers' mastery of the developing context of discourse anaphora and meanwhile discovery of the contribution made by the author. And above all, this organization (i.e. a literature review is followed by the author's theoretical model and the experiment) might be more coherent internally and externally, and to a certain extent might increase the readability of the book. Another point is concerned with the number of participants in the experiments, which include 20 native speakers of English and 20 native speakers of Chinese. The samples are comparatively small, which, to a certain extent, might influence the credibility of the research result. The last point is about the data for the text analysis in Chapter Five. This chapter presents the following criteria for choosing stories: length, point of view, role of character, and referential environment. Yet, another feature, generic structure, should be included as one of the criteria. "Genre represents the system of staged goal-oriented social processes through which social subjects in a given culture live their lives." (Christie \& Martin, 1997, p. 13) So, what generic structure expresses is the fundamental nature of a genre (i.e. narrative). Anyhow, even though it has a few minor weaknesses, this book is definitely well-written and is highly recommended. 
International Journal of Applied Linguistics \& English Literature

ISSN 2200-3592 (Print), ISSN 2200-3452 (Online)

Vol. 1 No. 5; September 2012 [Special Issue on General Linguistics]

\section{References}

Bussmann, Hadumod. (2000). Routledge dictionary of language and linguistics. Beijing: Foreign Language Teaching and Research Press.

Christie, F. \& Martin, J. R. (1997). Genre and institutions: Social processes in the workplace and school. London: Cassell.

Clark, Robin \& Parikh, Prashant. (2007). Game theory and discourse anaphora. Journal of Logic, Language and Information. 16 (3): 265-282. Kluwer Academic Publishers Hingham, MA, USA.

Huang, Yan. (2000). Discourse anaphora: Four theoretical Models. Journal of Pragmatics. (32):151-176.

Reuland, Eric, Everaert, Martin, \& Volkova, Anna, (2011), Anaphora. [Online] Available:

http:/oxfordbibliographiesonline.com/view/document/obo-9780199772810/obo-9780199772810-0050.xml

Wikipedia. [Online] Available: http://en.wikipedia.org/wiki/Literature_review

\section{Notes}

This review appeared originally in the LINGUIST List at http://linguistlist.org/issues/15/15-839.html. 